\title{
ELLIPTIC INTEGRALS IN TERMS OF LEGENDRE POLYNOMIALS
}

\author{
by M. O. GONZÁLEZ \\ (Received 13th April, 1954)
}

1. Consider the elliptic integral of the first kind

or, alternatively,

$$
u=\operatorname{sn}^{-1} x=\int_{0}^{x} \frac{d x}{\sqrt{\left(1-x^{2}\right)\left(1-k^{2} x^{2}\right)}}
$$

$$
u=F(\phi, k)=\int_{0}^{\phi} \frac{d \phi}{\sqrt{1-k^{2} \sin ^{2} \phi}}
$$

where $x=\sin \phi$. It is customary in the theory of elliptic integrals to let $k=\sin \alpha, k^{\prime}=\cos \alpha$, so that $k^{\prime 2}+k^{2}=1$. For convenience we shall also introduce the parameter

$$
\lambda=k^{\prime 2}-k^{2} \text {. }
$$

The substitution $x=\sin \phi$ is equivalent to

$$
x=\frac{2 v}{1+v^{2}}
$$

where $v=\tan \frac{\phi}{2} . \quad$ By means of this substitution we find that

$$
u=2 \int_{0}^{v} \frac{d v}{\sqrt{1+2 \lambda v^{2}+v^{4}}}
$$

We have

$$
\left(1+2 \lambda v^{2}+v^{4}\right)^{-\frac{1}{1}}=\sum_{n=0}^{\infty} P_{n}(-\lambda) v^{2 n}=\sum_{n=0}^{\infty}(-1)^{n} P_{n}(\lambda) v^{2 n}
$$

where $P_{n}(\lambda)$ denotes the Legendre polynomial of order $n$. This series converges uniformly with respect to $v$ and $\lambda$ when $|\lambda| \leqslant a,|v| \leqslant b, a$ and $b$ being positive constants such that $2 a b^{2}+b^{4} \leqslant 1-\delta(\delta>0)$.

Hence it follows that

$$
u=2 \sum_{n=0}^{\infty} \frac{(-1)^{n}}{2 n+1} P_{n}(\lambda) v^{2 n+1}
$$

or

$$
u=2 \sum_{n=0}^{\infty} \frac{(-1)^{n}}{2 n+1} P_{n}(\lambda) \tan ^{2 n+1} \frac{\phi}{2} .
$$

For $\phi=\frac{\pi}{2}$ we find formally

$$
K=2 \sum_{n=0}^{\infty} \frac{(-1)^{n}}{2 n+1} P_{n}(\lambda)
$$


a formula which we shall establish rigorously in ( $\$ 2$ ). If, in addition, $\lambda=1$, the formula above gives

$$
\frac{\pi}{4}=\sum_{n=0}^{\infty} \frac{(-1)^{n}}{2 n+1}=1-\frac{1}{3}+\frac{1}{5} \cdots
$$

Thus (3) is an extension of the Gregory-Leibnitz series.

2. Series expansions for $K, K^{\prime}, E$ and $E^{\prime}$.

It is well known that the complete elliptic integral of the first kind $K$ can be expressed in terms of Gauss's hypergeometric function as follows

$$
K=\frac{\pi}{2} F\left(\frac{1}{2}, \frac{1}{2}, 1 ; k^{2}\right)
$$

On the other hand, the Legendre function of degree $n$ of the first kind is defined by means of the equation

$$
P_{n}(\lambda)=F\left(-n, n+1,1 ; \frac{1-\lambda}{2}\right)
$$

which, for $n=-\frac{1}{2}$, gives

$$
P_{-1 / 2}(\lambda)=F\left(\frac{1}{2}, \frac{1}{2}, 1 ; \frac{1-\lambda}{2}\right)
$$

Since $\frac{1-\lambda}{2}=k^{2}$, from (4) and (5) we obtain

$$
K=\frac{\pi}{2} P_{-1 / 2}(\lambda) .
$$

Similarly,

$$
K^{\prime}=\frac{\pi}{2} P_{-1 / 2}(-\lambda)
$$

Also, since

$$
E=\frac{\pi}{2} F\left(-\frac{1}{2}, \frac{1}{2}, 1 ; k^{2}\right)
$$

and

it can be shown that

$$
E^{\prime}=\frac{\pi}{2} F\left(-\frac{1}{2}, \frac{1}{2}, 1 ; k^{\prime 2}\right)
$$

$$
E=\frac{\pi}{4}\left[P_{-1 / 2}(\lambda)+P_{1 / 2}(\lambda)\right]
$$

and that

$$
E^{\prime}=\frac{\pi}{4}\left[P_{-1 / 2}(-\lambda)+P_{1 / 2}(-\lambda)\right] .
$$

Now, if we expand the Legendre function $P_{-1 / 2}(\lambda)$ in a series of Legendre polynomials we obtain

$$
P_{-1 / 2}(\lambda)=\frac{4}{\pi} \sum_{n=0}^{\infty} \frac{(-1)^{n}}{2 n+1} P_{n}(\lambda)
$$

Similarly, by expanding $P_{1 / 2}(\lambda)$ in a series of Legendre polynomials we obtain

$$
P_{1 / 2}(\lambda)=\frac{4}{\pi} \sum_{n=0}^{\infty}(-1)^{n+1} \frac{2 n+1}{(2 n-1)(2 n+3)} P_{n}(\lambda)
$$


Whence,

$$
\begin{aligned}
K & =2 \sum_{n=0}^{\infty} \frac{(-1)^{n}}{2 n+1} P_{n}(\lambda), \quad \ldots \ldots \ldots \ldots \ldots \ldots \ldots \ldots \ldots \ldots \ldots \ldots \ldots \ldots \ldots \ldots \ldots \ldots \ldots \ldots \ldots \\
K^{\prime} & =2 \sum_{n=0}^{\infty} \frac{1}{2 n+1} P_{n}(\lambda), \quad \ldots \ldots \ldots \ldots \ldots \\
E & =4 \sum_{n=0}^{\infty} \frac{(-1)^{n+1}}{(2 n-1)(2 n+1)(2 n+3)} P_{n}(\lambda), \\
E^{\prime} & =-4 \sum_{n=0}^{\infty} \frac{1}{(2 n-1)(2 n+1)(2 n+3)} P_{n}(\lambda) .
\end{aligned}
$$

For $\lambda=1$ it follows from (14) that

$$
\overline{8}=\sum_{n=0}^{\infty} \frac{(-1)^{n+1}}{(2 n-1)(2 n+1)(2 n+3)} .
$$

3. Series expansions for $\mathrm{cn}^{-1} x$ and for $\wp^{-1}(x)$.

By letting $x=\left(1-v^{2}\right) /\left(1+v^{2}\right)$ it can be shown that

$$
\operatorname{cn}^{-1} x=\int_{x}^{1} \frac{d x}{\sqrt{\left(1-x^{2}\right)\left(k^{\prime 2}+k^{2} x^{2}\right)}}=2 \sum_{n=0}^{\infty} \frac{(-1)^{n}}{2 n+1} P_{n}(\lambda)\left(\frac{1-x}{1+x}\right)^{n+1} \text {. }
$$

Also, by letting $x=e_{1}+\gamma^{2} u^{-2}$, where $3 e_{1}=2 \lambda \gamma^{2}, g_{2}=12 e_{1}^{2}-4 \gamma^{4}$, it is easy to verify that

$$
\wp^{-1}(x)=\int_{x}^{\infty} \frac{d x}{\sqrt{4 x^{3}-g_{2} x-g_{3}}}=\sum_{n=0}^{\infty} \frac{(-1)^{n}}{2 n+1} \gamma^{2 n} P_{n}(\lambda)\left(x-e_{1}\right)^{-(n+\downarrow)} \text {. }
$$

4. Expansion for the elliptic integral of the second kind.

Since

$$
E(\phi, k)=\frac{k^{2} \sin \phi \cos \phi}{\sqrt{1-k^{2} \sin ^{2} \phi}}+k^{\prime 2}\left[k \frac{\partial F}{\partial k}+F\right]
$$

we obtain, after introducing the parameter $\lambda$, and making use of (2) :

$$
E(\phi, \lambda)=\frac{(1-\lambda) \tan \phi}{\sqrt{4+2(1+\lambda) \tan ^{2} \phi}}+\sum_{n=0}^{\infty} \frac{(-1)^{n}}{2 n+1}\left[2\left(\lambda^{2}-1\right) P_{n}^{\prime}(\lambda)+(\lambda+1) P_{n}(\lambda)\right] \tan 2 n+1 \frac{\phi}{2},
$$

or, alternatively,

$$
E(\phi, \lambda)=\frac{(1-\lambda) \tan \phi}{\sqrt{4+2(1+\lambda) \tan ^{2} \phi}}+\sum_{n=0}^{\infty} \frac{(-1)^{n}}{2 n+1}\left[((2 n+1) \lambda+1) P_{n}(\lambda)-2 n P_{n-1}(\lambda)\right] \tan ^{2 n+1} \frac{\phi}{2}
$$

since

$$
\left(\lambda^{2}-1\right) P_{n}^{\prime}(\lambda)=n \lambda P_{n}(\lambda)-n P_{n-1}(\lambda)
$$

\section{UNIVERSTTY OF ALABAMA,}

U.S.A. 